\title{
Research on application of artificial intelligence technology in fire alarm system of substations
}

\author{
Rong Cheng ${ }^{1, *}$, Linjie Chai ${ }^{1}$ and Shiyao $\mathrm{Hu}^{1}$ \\ ${ }^{1}$ State Grid Hebei Economic Research Institute, Shijiazhuang, Hebei, 050000, China
}

\begin{abstract}
It is very important to identify fires in substations. Traditional fire alarm systems have problems such as low sensitivity and slow response. This article introduces the working principle of a fire alarm system based on artificial intelligence. And this article also designs different smoke particle sampling schemes for different areas in the substation, such as functional rooms/cable trenches, to ensure the correct operation of the system combined with the actual conditions of each area. Resulting that the fire alarm system based on artificial intelligence can detect the fire at the early stage and alarm in time.
\end{abstract}

\section{Background}

There are a large number of computers, power supplies, and expensive equipment, wires and cables, and various storage media in the substation. Among them, the components inside the equipment and the cable insulation sheath mostly use flammable materials such as phenolic fiber and polyvinyl chloride, which are very easy to burn and cause fires. In addition, various storage media such as paper, magnetic disks, and tapes are also important factors that cause fires.

With the development of science and technology, the integration of electronic products is getting higher and higher, and the size is getting smaller and smaller, resulting in higher potential fire hazards in the unit space. The equipment that was originally placed in multiple rooms is also all concentrated in one room now. The fire will have a more serious impact on computers, databases and other facilities and their operations. In addition, due to the high integration of equipment, the requirements for the operating environment are getting higher and higher, and any changes in temperature and humidity will cause the components to heat up. According to statistics, among the various causes of fires, 32\% of fires are caused by power supply systems (AC and DC power supplies, batteries, generators and power supply lines, etc.). $18 \%$ of fires are caused by other electrical equipment in the building, including air conditioning, heating equipment, lighting system, etc. $10 \%$ of fires are directly caused by wiring inside the equipment. When fire occurs, it will not only cause direct damage to the equipment, but also cause long-term harm to the surrounding equipment and items, since the generated gas from the electronic equipment of special material having a strong corrosive combustion.

Therefore, the substation is equipped with an intelligent auxiliary control system including the fire alarm system to discover and deal with fire hazards in time. The fire alarm control system includes a detector and a fire extinguishing system. The fire extinguishing system includes fire detection pipe devices, gas storage type dry powder fire extinguishers and suspended dry powder fire extinguishers. The detector collects the characteristic gas concentration signal. When the gas concentration exceeds the set threshold, the control chip outputs an alarm command to activate the fire alarm device, and at the same time, it starts to extinguish the fire.

\section{The shortcomings of traditional fire alarm systems}

At present, the commonly used fire alarm methods include manual inspections, point-type smoke detectors, line-type beam detectors, flame detectors, image detectors, etc. Among them, manual inspection has high accuracy but low efficiency, and inspectors are prone to fatigue during the inspection process. Point-type smoke detectors, line-type beam detectors and others have fixed sensitivity. Air conditioning systems are commonly used in substations, and the conventional air exchange rate of the air conditioning system is usually 15 to 60 times per hour, which will have a negative impact on detection. On the one hand, the smoke is greatly diluted make it difficult to reach the alarm threshold of the traditional equipment. On the other hand, the airflow make it difficult for smoke to reach the detector, cause alarm to delay or fail to report. Practice has proved that due to the influence of air conditioning system, equipment placement, room structure, traditional detectors often give an alarm after the fire has reached a certain scale and cannot report the exact location of the fire source.

\footnotetext{
*Corresponding author's e-mail: chengrong1991@126.com
} 
In terms of fire extinguishing systems, although carbon dioxide gas is not corrosive, and has a good protective effect in confined spaces. The high concentration of carbon dioxide gas released at low temperatures will also cause serious harm to electronic equipment and workers. Other gas fire extinguishing systems, such as FM200, IG541, will cause more or less adverse effects on the environment, objects and equipment after start up. And once started by mistake, it will cause a huge waste of fire extinguishing agent.

Based on the above analysis, the existing fire alarm system generally has the disadvantages of low sensitivity, damage to equipment and personnel after start up, and high cost of use. Therefore, there is an urgent need for a new generation of fire smoke detection equipment that is extremely sensitive, easy to maintain, and can detect and issue alarms in the early stages of a fire.

\section{Application of artificial intelligence technology in fire alarm system}

\subsection{Principle of aspirating smoke detection system based on artificial intelligence technology}

Aspirating smoke detection system based on artificial intelligence technology uses active detection and broadspectrum high-energy LED light source technologies. The suction pump draws air continuously, and analyze smoke particles. The system uses artificial intelligence technology to enable the detection equipment continuously learn changes in the environment and set the normal range of the environmental background smoke concentration. When the detector finds that the smoke concentration in the air is greater than the normal value, it will send out an alarm signal to extinguish the fire. The principle is shown in Figure 1.

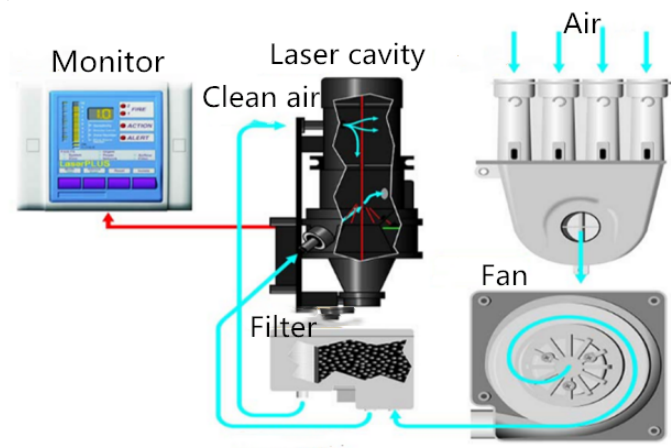

Figure 1. Principle of aspirating smoke detection system based on artificial intelligence technology

\subsubsection{Principle of air extraction system}

The principle of the air extraction system is shown in Figure 2, which mainly includes two methods: ceiling concealed installation and return air sampling. The concealed ceiling installation method is to install a hose in the ceiling of the house for sampling, and open a sampling hole the size of a dollar coin in the sampling pipe. This installation method is more beautiful for the pipes are invisible when you look up in the room, and this method is suitable for new substations. If it is installed on an exposed pipe under the ceiling, the advantage is that the construction is convenient and simple by simply open a sampling hole on the sampling pipe and no other accessories are needed. As shown on the left side of figure 3 , the white ring marks the position of the hole.

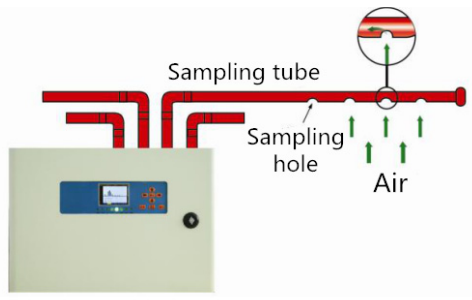

Figure 2. Principle of air extraction system
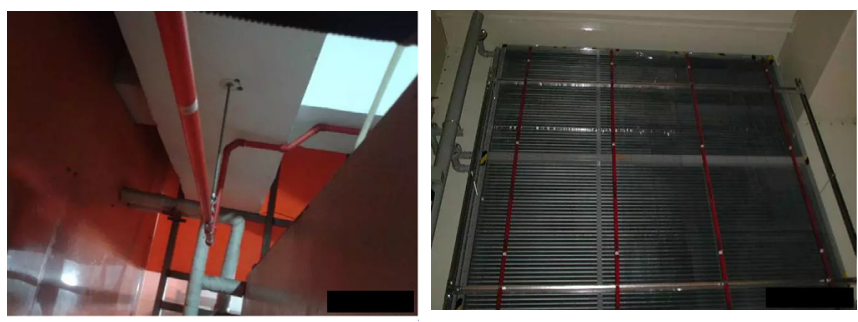

Figure 3. Diagram of ceiling concealed installation and return air sampling

Return air sampling is to arrange the sampling pipe at the return air outlet. Due to the air flow, the smoke will follow the airflow to the return air outlet, and the smoke can be collected and detected quickly. As shown on the right side of figure 3 , the red tube is the sampling tube, and the white ring is the sampling hole label.

\subsubsection{Smoke detection technology}

The aspirating smoke detector uses a broad-spectrum high-energy LED light source detection technology. The system uses broad-spectrum high-energy LED light source detection technology, by the scattering of smoke particles to the light source, the forward receiver detects the light addition ratio, and performs multi-band scanning of the smoke particles in the ignition phase of the fire, and then converts them into the smoke concentration, as shown in figure 4. The detection sensitivity of the broad-spectrum high-energy LED light source is as high as $0.001 \% \mathrm{obs} / \mathrm{m}$, and it is not sensitive to large-size particles such as dust and water mist. Therefore, the detector can have sufficient sensitivity to smoke particles produced by fire and effectively prevent false alarms.

The system has the advantages of strong environmental adaptability, simple structure, low cost, and easy maintenance. It can work under minus $30^{\circ} \mathrm{C}$ and dusty conditions. 


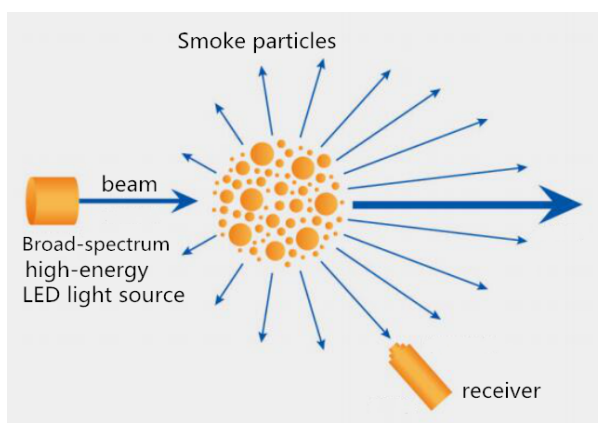

Figure 4. Diagram of broad-spectrum high-energy LED light source detection technology

\subsubsection{Application of artificial intelligence technology in the system}

Affected by the geographical environment of the substation location, the normal range of background smoke concentration has greater variability. For example, in northern China, the weather is relatively dry and the ambient smog concentration is larger, while in the south, it is smaller.

This system uses artificial intelligence technology and aspirating smoke detectors together, so that the detection equipment continuously learns the changes of the environment, and the alarm threshold setting takes into account the normal range of the environmental background smoke concentration, so that the alarm sensitivity is always maintained at an optimal level. The detector will not produce false alarms due to motivation of ambient smoke concentration caused by dust or other activities.

After the detector is used for a period of time, more and more dust will accumulate in the filter, which will reduce the efficiency of the filter and reduce the smoke particles entering the detection chamber. At this time, if there is no compensation, the sensitivity of the detector will be significantly reduced, and cause the filter blocked and the detector will not alarm. Artificial intelligence technology is used for automatic compensation. As the efficiency of the filter decreases, the alarm setting value of the detector will be adjusted and automatically reduced to ensure the sensitivity of the equipment.

\subsubsection{Material selection of detector sampling tube}

ABS sampling pipes should be used in places with corrosive gas and large temperature changes. When the pipeline is short and the total number of elbows is less than 4, UPVC material can be considered. If the pipeline is long, PVC pipe can be used, which can be manually bent to reduce air resistance. Pay attention, all materials must be flame retardant.

\subsection{Design scheme of fire alarm system based on artificial intelligence technology in a substation}

The substation contains functional room/secondary equipment room/battery room, etc., which need to be installed with fire alarm devices. This article intends to design a fire alarm system based on artificial intelligence technology and give the layout of each room.

\subsubsection{Sampling plan of function room}

The data room/tool room in the substation can be regarded as a function room. The air sampling in this type of room is generally arranged in a grid, as shown in the figure below. Considering that there are no flammable and explosive materials in this type of room, fire detection is relatively simple. The general principle is that the density of sampling holes is greater than that of traditional point-type smoke detectors.

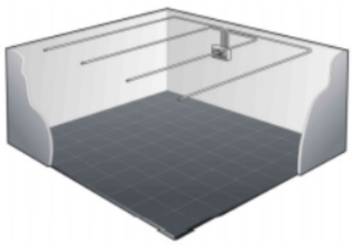

Figure 5. Sampling plan of function room

\subsubsection{Sampling plan of function room}

The main fire risks in the secondary equipment room include cable concentrated areas, high and low voltage switchgear and control equipment, etc. The typical ignition sources are: sparks and electrostatic discharge and overheated electrical control equipment, such as switchgear and cables. There are several difficulties in fire detection in such locations. When the cabinet is on fire, for the initial heat dissipation is not high, it will adversely affect the external detection of smoke. In addition, when the screen cabinet adopts forced ventilation, it may cause the smoke to be diluted, which limits the sensitivity of detection in the cabinet.

Therefore, the secondary equipment room needs to install both ceiling-type and cabinet-type sampling pipelines. Among them, the ceiling sampling is used in the indoor space, and the air sampling in the cabinet is used in the equipment screen cabinet. The design of sampling on the ceiling is similar to that of the functional room. The positioning of the piping system and the sampling hole depends on the layout and configuration of the equipment room. The spacing and density between the sampling holes should not be lower than the position of the traditional point-type smoke detector.

The sampling in the equipment cabinet is recommended to adopt the fully enclosed type or the top ventilation type. Top ventilation type is to install the sampling pipe directly on the ventilation grille of the electrical cabinet. Each electrical cabinet must have at least one dedicated sampling hole, and the sampling hole should directly face the airflow out of the cabinet. Sampling in the cabinet is to install the sampling pipe directly through the top of the electrical cabinet or through the bottom of the electrical cabinet from the gap between the floors. As shown in figure 6 . 


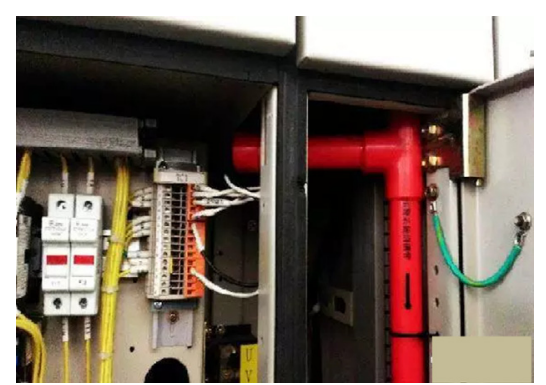

Figure 6. Sampling plan of the cabinet

\subsubsection{Sampling plan of cable trench}

The cable trench is used to lay communication, control and various power cables. The cable trench connects the control room, the prefabricated warehouse and the battery room, and is generally located below the ground. Due to the large amount of constant power on the cable, the cable trench has a great fire hazard.

In cable trenches, the most effective way to detect is to install sampling pipes above $10 \%$ of the trench height under the cable trench cover, as shown in Figure 7. Such a uniform arrangement can promptly discover hidden fire hazards in various places in the cable trench.

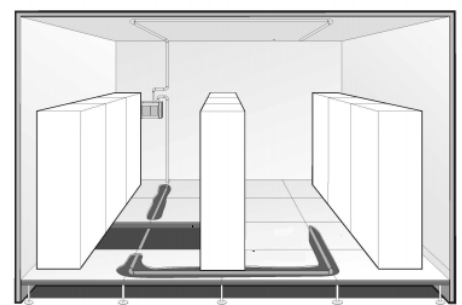

Figure 7. Sampling plan of cable trench

\subsubsection{Sampling plan of battery room}

The battery room is equipped with lead-acid or nickelcadmium batteries that supply power to the UPS. Due to the accumulation of high-concentration hydrogen, the battery room may explode. Generally, a continuously operating controlled fan is installed in the battery room to extract the accumulated hydrogen and provide ventilation.

Therefore, the exhaust sampling device can be installed at the exhaust grille of the air duct or positioned inside the air duct for sampling, As shown below. Early smoke generally tends to follow natural air currents. Positioning the pipe with sampling holes on the air grille of the exhaust ventilation system can detect the smoke generated in the battery room at an early stage.

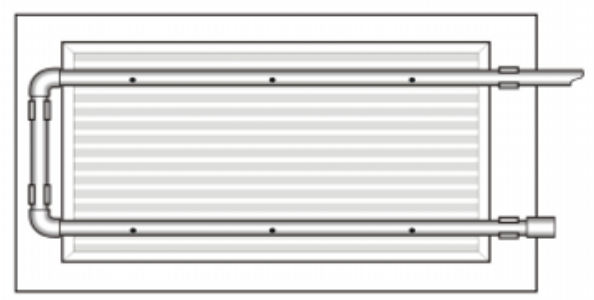

Figure 8. Sampling plan of battery room

\section{4 conclusion}

The fire alarm system based on artificial intelligence technology deeply combines aspirating smoke detectors with AI technology, and can rely on the support of big data to achieve high sensitivity and low false alarm rate for fire prevention. The system uses broad-spectrum high-energy LED light source technology, and the air pump uninterruptedly pumps air to analyze the smoke particles. By using artificial intelligence technology, the detection equipment continuously learns the changes of the environment, and sets the range of the environmental background smoke concentration. When the detector finds that the concentration of smoke particles in the air is greater than the normal value, it will send out an alarm signal to extinguish the fire. By designing different smoke particle sampling schemes for different areas in the substation, such as functional rooms/cable trenches, the correct operation of the system can be ensured.

\section{References}

1. Xin Guan, Hong Miao, Huichuan Zhang. (2005) Intelligent fire alarm and control system. Journal of Shenyang University of Technology, 27: 312-316

2. Zhen Liu. (2018) Design of fire alarm system. Electric technology, 10: 29

3. Yubao Liu, Zhibin Mei, Wenqing Wang, Linlin Cheng. (2005) Simulation on the sampling pipe network of aspirating smoke detector. Fire safety science, 14: 154-159

4. IEI. VESDA E07-D Intelligent Addressable HSSD Detector Operator's handbook[M]. IEI, 1992:1-4

5. Lirui Cao. (2014) Problems and countermeasures in the application of very early aspirating smoke detection system. Neimenggu Technology and Economy, 2: 114-115

6. Qian Zhang. (2015) Application Analysis of Aspirating Smoke Detector in Hangzhou Metro. Science and wealth, 15: 119

7. Hang Qi. (2015) Research on Application of High Sensitive Aspiration Smoke Detection and Alarm Technology. Electronic Technology and Software Engineering, 21: 125 